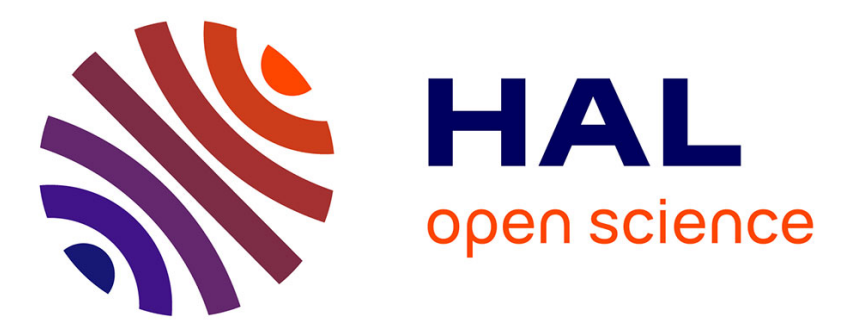

\title{
On the nontrivial wave-vector dependence of the elastic modulus of glasses
}

Giacomo Baldi, Valentina M. Giordano, Beatrice Ruta, Giulio Monaco

\section{To cite this version:}

Giacomo Baldi, Valentina M. Giordano, Beatrice Ruta, Giulio Monaco. On the nontrivial wave-vector dependence of the elastic modulus of glasses. Physical Review B: Condensed Matter and Materials Physics (1998-2015), 2016, 93, pp.144204. 10.1103/PhysRevB.93.144204 . hal-02304937

\section{HAL Id: hal-02304937 \\ https://univ-lyon1.hal.science/hal-02304937}

Submitted on 16 Feb 2021

HAL is a multi-disciplinary open access archive for the deposit and dissemination of scientific research documents, whether they are published or not. The documents may come from teaching and research institutions in France or abroad, or from public or private research centers.
L'archive ouverte pluridisciplinaire HAL, est destinée au dépôt et à la diffusion de documents scientifiques de niveau recherche, publiés ou non, émanant des établissements d'enseignement et de recherche français ou étrangers, des laboratoires publics ou privés. 
PHYSICAL REVIEW B 93, 144204 (2016)

\title{
On the nontrivial wave-vector dependence of the elastic modulus of glasses
}

\author{
Giacomo Baldi* \\ IMEM-CNR, Parco Area delle Scienze 37/A, I-43124 Parma, Italy \\ Valentina M. Giordano \\ Institute of Light and Matter, UMR5306 Université Lyon 1-CNRS, Université de Lyon, F-69622 Villeurbanne Cedex, France; \\ Université Grenoble Alpes, SIMAP, F-38000 Grenoble, France; \\ and CNRS, SIMAP, F-38000 Grenoble, France \\ Beatrice Ruta \\ ESRF-The European Synchrotron, CS 40220, F-38043 Grenoble Cedex 9, France \\ Giulio Monaco \\ Dipartimento di Fisica, Università di Trento, I-38123 Povo (Trento), Italy \\ (Received 22 September 2015; revised manuscript received 25 March 2016; published 22 April 2016)
}

\begin{abstract}
Recent theoretical models for the vibrations in glasses assume that the complex elastic modulus depends on frequency but not on the wave vector, $q$. This assumption translates in a simple $q$ dependence of the dynamic structure factor, which can be experimentally tested. Following the suggestion of a recent paper [U. Buchenau, Phys. Rev. E 90, 062319 (2014)], we present here a new analysis, performed in $q$ space, of inelastic x-ray scattering data of supercooled silica. The outcome of the analysis is compared to the more common approach in the frequency domain and indicates that the mentioned theoretical assumption is consistent with the data only below the boson peak frequency. At higher frequencies it gives rise to a breakdown of the classical second moment sum rule. This violation arises from the underlying assumption of the presence of a single excitation in the spectra. A comparison with the vibrational dynamics of $\alpha$-cristobalite suggests, on the contrary, that in the terahertz frequency domain the inelastic spectrum of the glass gains contributions from both acousticlike and opticlike modes. A microscopic theory of the vibrations in glasses cannot neglect the medium range order in their structure, which gives rise to dispersion curves within a pseudo-Brillouin zone.
\end{abstract}

DOI: 10.1103/PhysRevB.93.144204

\section{INTRODUCTION}

Glass-forming liquids remain frozen in an out of equilibrium state when rapidly cooled below their glass transition temperature, $T_{g}$. The structural relaxation in the transition region is characterized by strongly correlated motion and by dynamical heterogeneities [1]. Neighboring atoms have similar mobility, which can differ considerably from that of more distant groups of atoms. When observed on a picosecond time scale both a glass and a supercooled liquid appear as vibrating around an average configuration, which is not strongly affected by the glass transition process, since the structural relaxation takes place on a time scale that is many orders of magnitude slower. For this reason it is commonly accepted that the terahertz vibrational dynamics of glasses or supercooled liquids is not markedly influenced by the structural relaxation, although some evidences of a direct relationship between the slow and fast dynamics have been reported in literature [2,3].

Recent numerical simulation studies have revealed that the elasticity of glasses is heterogeneous on the nanometer length scale [4-9], suggesting a possible relation with the dynamical heterogeneities above $T_{g}$. In particular, a correlation length of tens of interatomic distances has been revealed in the nonaffine displacement field of various simulated glasses

\footnotetext{
*giacomo.baldi@cnr.it
}

under an external perturbation [4,5]. Numerical simulations of polymeric glasses [6,7] and of Lennard-Jones and softsphere systems [8,9] indicate that the local elastic modulus is heterogeneous and the width of its distribution increases as the length scale over which it is computed is reduced, implying that on the macroscopic scale these fluctuations are averaged out. However, at present, the numerical works do not indicate a length scale for the heterogeneities, although there have been indications for a fractal-like distribution of the local elastic modulus [7]. On the experimental side, evidences of elastic heterogeneities are still scarce [10,11].

The terahertz vibrational dynamics of glasses is marked by an excess of vibrational states over the Debye prediction, called the boson peak (BP). Below the BP frequency the mean-free path of the sound waves follows the Rayleigh scattering law, being proportional to the inverse of the fourth power of frequency [12-17]. Various theoretical models for the vibrations in glasses are based on the idea of the presence of elastic heterogeneities. The theory of Schirmacher and coworkers $[18,19]$ assumes a Gaussian distribution of the local elastic modulus and predicts the frequency dependence of the macroscopic modulus. This frequency dependence gives rise to a $\mathrm{BP}$ feature in the density of vibrational states. A different theory [20,21], based on the jamming scenario and on an effective medium approximation, describes the peculiar features of the vibrational dynamics of glasses as arising from the proximity to an elastic instability. In both theories the complex elastic modulus depends on frequency 
but not on the wave vector, $q$, giving rise to a simple $q$ dependence of the dynamic structure factor, which can be tested on the experimental data, as suggested by Buchenau [22]. Recent works have given experimental evidence of the close correspondence between the BP in glasses and the first Van Hove singularity in the crystalline forms of similar mass density [23-25]. Such an interpretation of the origin of the $\mathrm{BP}$ is radically different from the one advanced by the above-mentioned theoretical approaches [18-21].

In the present paper we address the validity of the hypothesis of a $q$-independent elastic modulus, in order to determine the frequency range where such an approximation can be considered appropriate. To this aim we describe a normalization procedure that allows one to determine the $q$ dependence of the spectra at fixed frequency from a set of fixed $q$ frequency scans. The procedure relies only on measured quantities, without making use of any sum rule. We apply the method to inelastic x-ray scattering (IXS) data of silica in the supercooled state $(T=1620 \mathrm{~K})$ and we obtain the following result: a single excitation model violates the classical second moment sum rule above the BP frequency. In order to explain this observation we compare the single excitation model with a previously published analysis with two excitations and with recent data for the vibrational dynamics of $\alpha$-cristobalite, the crystalline polymorph with the closest mass density to the one of vitreous silica. Details on the IXS experiment on supercooled silica can be found in a previous paper [15], where those data have been partially published. We have chosen this data set because of the high signal-to-noise ratio of the spectra and because they were measured on a fine grid in $q$.

The paper is organized as follows. In Sec. II we describe the normalization procedure and we model the spectra with the dynamic structure factor that one obtains under the hypothesis of a $q$-independent elastic modulus. Section III is devoted to a comparison of this analysis to the more common approach in the frequency domain, where the IXS spectra are described in terms of a damped harmonic oscillator model (DHO). To this aim we have extended the previously published analysis to the high- $q$ range. Moreover, the normalization procedure allows us to obtain new information, namely the DHO intensity in absolute units. We will show that both approaches are unable to properly describe the measured spectra at frequencies larger than the BP one. Finally, in Sec. IV, we compare the analysis of the spectra in terms of a single- and a double-excitations model and we discuss the relationship between the dynamics of the glass and that of the $\alpha$-cristobalite single crystal. This analysis shows the inadequacy of models that do not take into account the modes dispersion induced by the structural order on the medium range.

\section{DATA ANALYSIS}

\section{A. Normalization procedure}

The observable quantity in an IXS experiment is the intensity, $I(q, \omega)$, scattered by the sample at an exchanged wave vector $q$ and frequency $\omega$, where $\omega=0$ corresponds to elastic scattering. The measured intensity can be written in terms of the dynamic structure factor, $S(q, \omega)$, as:

$$
I(q, \omega)=I_{0}(q) \int_{-\infty}^{\infty} d \omega^{\prime} S\left(q, \omega^{\prime}\right) R\left(\omega-\omega^{\prime}\right)+y_{0},
$$

where $R(\omega)$ is the instrumental resolution function in angular frequency, $I_{0}(q)$ is a wave-vector-dependent normalization function and $y_{0}$ is the background. This equation is strictly valid only for monatomic systems, because the contributions of the different atoms of a polyatomic sample are weighted by their atomic form factors. In the following we will make the approximation that this expression is valid also for polyatomic systems.

IXS beam lines are normally operated to measure the scattered intensity as a function of frequency at a fixed exchanged wave vector, since the frequency scan is routinely performed by varying the temperature of the main monochromator. In principle, it is possible [26] to perform the measurement at a fixed frequency as a function of $q$, but this procedure encounters the technical difficulty of maintaining the temperature stability of the monochromator and of the analyzers during the time requested to acquire a spectrum.

Different approaches can be used to determine the function $I_{0}(q)$ of Eq. (1) in order to obtain the $q$ dependence from a set of fixed- $q$ spectra. A first possibility is to use a reference scatterer, a sample for which the relation between $I(q, \omega)$ and $S(q, \omega)$ is well known. In the case of IXS, however, such a reference sample is not well established. A second class of approaches makes use of the sum rules to determine $I_{0}(q)$. The simplest method is to use the zeroth-order moment and to normalize the spectra to the static structure factor, $S(q)$, which can be derived by integration of Eq. (1), assuming that $R(\omega)$ is normalized to unity:

$$
\int_{-\infty}^{\infty} d \omega\left[I(q, \omega)-y_{0}\right]=I_{0}(q) S(q) .
$$

This approach is not straightforward because it requires $S(q)$ to be known with good accuracy in the same $q$ range of the experiment. Moreover, $S(q)$ can vary between different samples with different thermal histories and should thus be measured on the same sample. Another possibility is the use of the first moment sum rule, which, however, is helpful only if the temperature is sufficiently low that the intensities of the Stokes and anti-Stokes peaks differ significantly [27].

In principle, a good way to normalize the spectra also at high temperatures is given by the classical second moment sum rule [22,28-30], but the use of this normalization procedure on the experimental data is of difficult implementation for two main reasons. One relates to the fact that the spectrum is the convolution of the dynamic structure factor with the resolution function, which has Lorentzian-like tails and thus its second moment is, strictly speaking, undefined. The second big difficulty arises because the spectra are collected in a limited frequency window, while a proper determination of the second moment would require precise measurements at high frequencies, where this is difficult.

For the aforementioned reasons we followed a different approach to determine $I_{0}(q)$. This quantity can be written as (see the supplemental material of Ref. [24] for more details):

$$
I_{0}(q)=\bar{I}_{0} \eta(q) \sigma(q)\left(\hat{\epsilon}_{i} \cdot \hat{\epsilon}_{f}\right)^{2} \frac{1}{N}\left|\sum_{l=1}^{N} F_{l}(q)\right|^{2},
$$

where $\bar{I}_{0}$ is a multiplicative constant, $\eta(q)$ accounts for the analyzer reflectivity and for the efficiency of the detector, $\sigma(q)$ 
is a term accounting for absorption effects and is a function of the absorption coefficient, $\mu$, and of the sample geometry, $\hat{\epsilon}_{i}$ and $\hat{\epsilon}_{f}$ are the polarization vectors of the incoming and outgoing X-ray beams, $N$ is the number of atoms per molecule, and $F_{l}(q)$ is the atomic form factor of atom $l$ in the molecule. The quantity $\sigma(q)$ for a flat sample of thickness $L$ is

$$
\sigma(q)=\frac{e^{\mu L\left(1-\frac{1}{\cos \theta}\right)}-1}{\mu L\left(1-\frac{1}{\cos \theta}\right)},
$$

where $\theta$ is the scattering angle. It is worth noting that the efficiency, $\eta$, of a single analyzer-detector couple is independent of $q$. The ID16 beam line being equipped with a nine-analyzer chamber, which allowed us to measure nine spectra at different scattering angles at the same time, it is necessary to measure the relative efficiency of the analyzer-detector couples with respect to a reference one (more details on the experimental setup can be found in Ref. [15]). We performed this task by measuring the elastic line of a plexiglass sample at $q=10 \mathrm{~nm}^{-1}$ using each analyzer.

The followed approach allows us to determine $I_{0}(q)$ up to a multiplicative constant $\bar{I}_{0}$, which can be obtained by comparing the static structure factor from Eq. (2) with the $S(q)$ from the literature, as shown in Fig. 1(a). Note that the value of $\bar{I}_{0}$ affects only the intensity of the spectra but not their $q$ dependence. The figure allows one to appreciate the good agreement between the $S(q)$ determined from the IXS data and that obtained from diffraction, proving the reliability of the normalization procedure.

\section{B. Model}

The normalization procedure outlined in the previous section allows us to obtain, in absolute units, a quantity equal to the convolution of the dynamic structure factor with the instrumental resolution function, plus an almost negligible background arising from the detectors noise.

The $S(q, \omega)$ in one phonon approximation can be written in terms of its classical counterpart as [16,32]:

$$
S(q, \omega)=\frac{\hbar \omega / k_{B} T}{1-e^{-\hbar \omega / k_{B} T}} S^{c l}(q, \omega),
$$

where $k_{B}$ is the Boltzmann constant and $T$ the temperature, and

$$
S^{c l}(q, \omega)=S(q) f(q) \delta(\omega)+\frac{k_{B} T q^{2}}{\pi m \omega} \operatorname{Im}\left\{G_{L}(q, \omega)\right\} .
$$

The first term to the right-hand side of the expression, proportional to a Dirac $\delta$ function, $\delta(\omega)$, accounts for the density fluctuations that are frozen below the glass transition temperature and contribute only to the elastic scattering. Their weight is proportional to the nonergodicity parameter $f(q)$, defined as the ratio of the elastic to the total intensity. The second term contains the imaginary part of the longitudinal Green's function $G_{L}$, while $m$ is the average atomic mass.

The Green's function can be expressed in terms of a self-energy $\Sigma=\Sigma(q, \omega)$, with the tacit assumption that the spectrum has a single inelastic peak [16]:

$$
G_{L}(q, \omega)=\frac{1}{\omega^{2}-q^{2} \Sigma(q, \omega)} .
$$

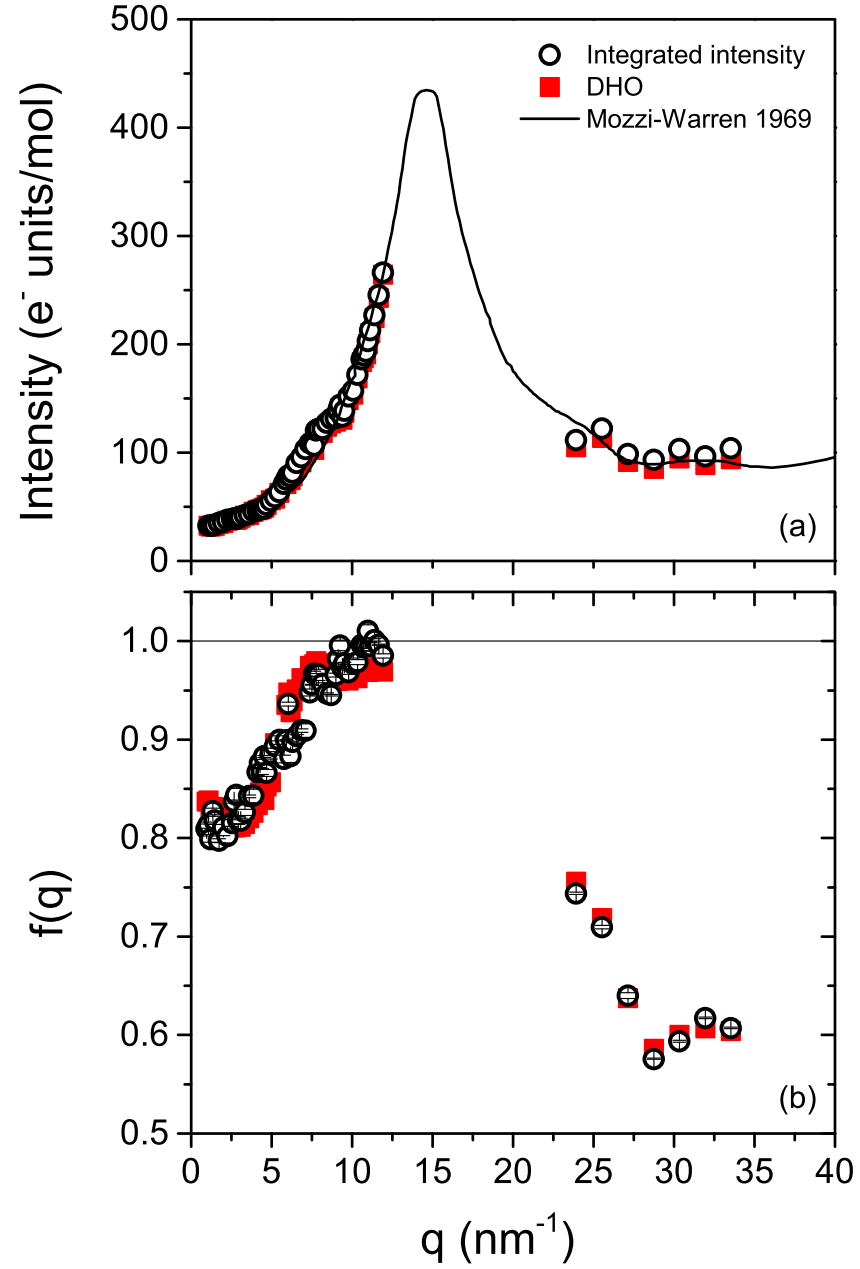

FIG. 1. (a) Diffraction intensity from reference [31] (continuous line) compared to the integrated intensity of the IXS spectra normalized in absolute units (black circles) and to the total intensity of the DHO model fitted to the spectra (red squares). The plotted quantity is: $\frac{1}{N}\left|\sum_{l} F_{l}(q)\right|^{2} S(q)$, in the notation of Eq. (3). (b) Nonergodicity parameter, $f(q)$, determined from the elastic line intensity in Eq. (6) as explained in the text (black circles) and from the fit of the entire spectrum to the DHO model (red squares). The horizontal line is set at $f=1$, its maximum value.

A common approximation to the self-energy is the one leading to the damped harmonic oscillator model (DHO) [16]:

$$
\Sigma_{\mathrm{DHO}}(q, \omega)=\frac{\Omega^{2}(q)}{q^{2}}+i \frac{\omega \Gamma(q)}{q^{2}} .
$$

In this expression $\Omega$ is the peak position in the current correlation function and $\Gamma$ is a parameter related to the width of the peak (it is the full width at half maximum of the peak in the limit $\Gamma / \Omega \rightarrow 0$ ). The self-energy for the DHO has a trivial frequency dependence so that it is commonly used to analyze inelastic spectra measured at a fixed $q$. It is worth noting that the DHO has a nonzero value at $\omega=0$, so that a determination of the parameter $f(q)$ in Eq. (6) requires a fit to the entire spectrum, including the elastic and the inelastic components. The fourth parameter of the DHO model is $S(q)$.

The IXS data of vitreous silica that we discuss in the present manuscript were analyzed in Ref. [15] by making use of a 


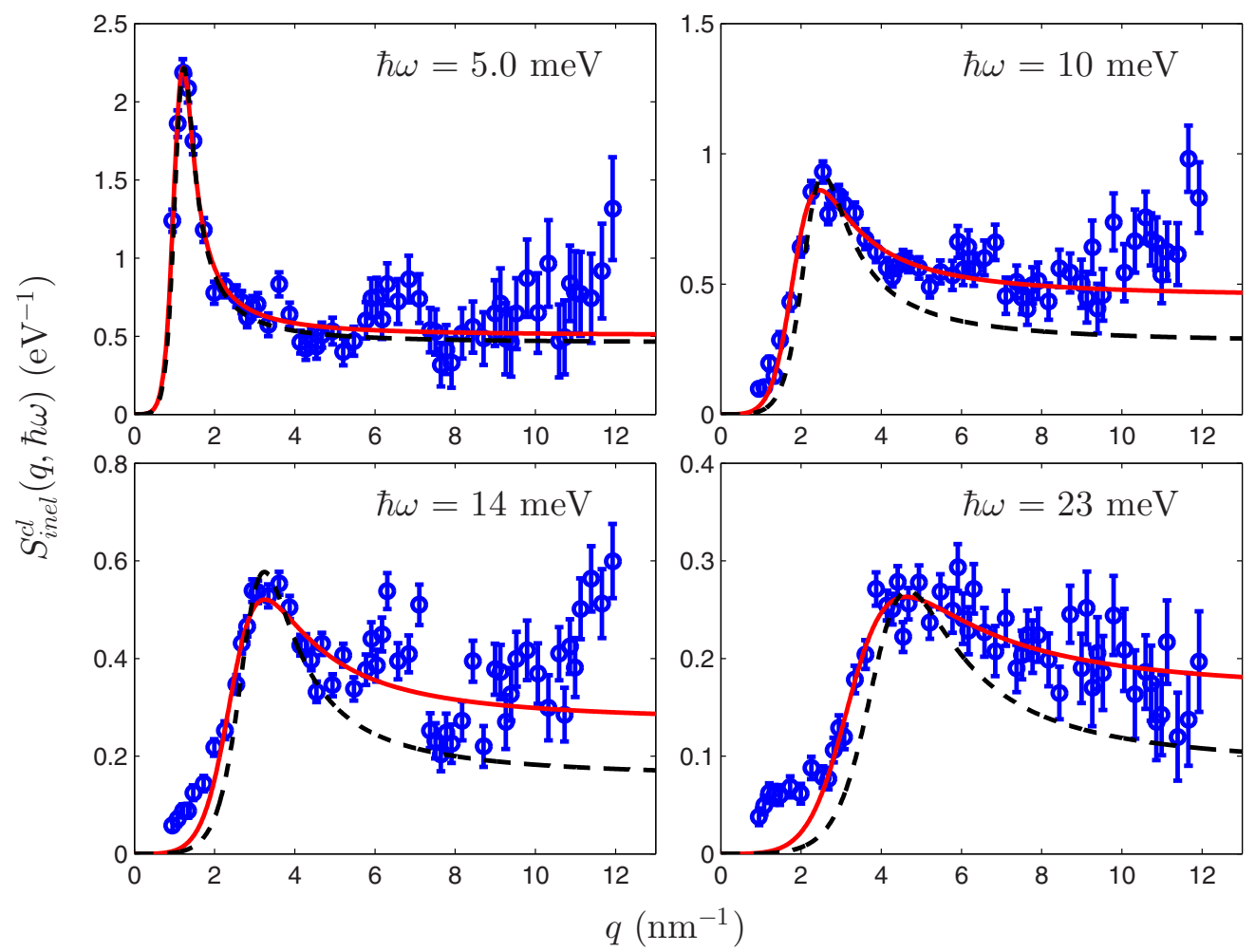

FIG. 2. Selection of inelastic spectra as a function of $q$ at the indicated frequencies. The continuous lines (red) are the best fitting curves obtained with the model based on a $q$-independent self-energy and a free parameter $A$ as defined in Eq. (10). The dashed (black) curves correspond to the fit with the constraint of $A=1$.

single DHO function for the inelastic peaks. In that work we focused our attention to the low- $q$ range $\left(q<4 \mathrm{~nm}^{-1}\right)$, a region where a single-excitation model provides a reliable description of the spectra. In the following we will present a comparison between the analysis of the spectra at fixed $\omega$ with the one at fixed $q$ and to this aim we will also extend the DHO analysis to the entire range of measured wave vectors (the dots in Fig. 1).

The common hypothesis made in the theories mentioned in the introduction [18-21] is to drop the $q$ dependence of the self-energy, assuming:

$$
\Sigma=\Sigma(\omega)=\Sigma^{\prime}(\omega)+i \Sigma^{\prime \prime}(\omega),
$$

where $\Sigma^{\prime}$ and $\Sigma^{\prime \prime}$ denote, respectively, its real and imaginary part. It is worth noting that $M=\rho \Sigma(\omega)$, with $\rho$ the mass density, is the frequency-dependent macroscopic longitudinal modulus, which is a complex quantity.

We begin by noting that the second term in Eq. (6), under the assumption of a $q$-independent self-energy, is purely inelastic for a harmonic glass, because at low frequency the damping is dominated by Rayleigh scattering and $\Sigma^{\prime \prime} \sim \omega^{3}$. We can thus obtain the inelastic spectra by subtracting an elastic line proportional to the instrumental resolution function. The proportionality factor is determined by a $\chi^{2}$ minimization routine including only a small range, $\pm 0.4 \mathrm{meV}$, around $\omega=0$. This procedure allows also to determine the nonergodicity parameter $f(q)$, which follows the behavior of the $S(q)$, as shown in the bottom panel of Fig. 1. The parameter $f$ reaches the value of one, within its uncertainty, for the highest $q$ s measured below the structure factor peak, meaning that the inelastic intensity in this range is very small compared to the elastic one. The squares (red) in the figure correspond to the parameter $f(q)$ determined from the analysis with a single DHO model and show a similar trend, although these points remain always below one because the DHO has the tendency to overestimate the inelastic intensity at the center of the spectrum [15].

A selection of inelastic spectra at fixed frequencies is reported in Fig. 2. These spectra are obtained by averaging the Stokes and anti-Stokes sides and performing an average on neighboring frequency points to improve the signal-to-noise ratio. Equation (9) together with the previous equations gives the following expression for the classical inelastic dynamic structure factor:

$$
S_{\text {inel }}^{c l}(q, \omega)=A(\omega) \frac{k_{B} T}{\pi m \omega} \frac{q^{4} \Sigma^{\prime \prime}(\omega)}{\left[\omega^{2}-q^{2} \Sigma^{\prime}(\omega)\right]^{2}+\left[q^{2} \Sigma^{\prime \prime}(\omega)\right]^{2}},
$$

where we added a multiplicative constant $A$ as a free parameter, in line with what is suggested in Ref. [22]. Consequently the model has four free parameters: $f(q), A(\omega), \Sigma^{\prime}(\omega)$, and $\Sigma^{\prime \prime}(\omega)$, the same number of parameters as the DHO model. It is in fact similar to a DHO, being the structure factor of a damped longitudinal phonon in $q$ space [22].

The best fitting curves, obtained by minimizing the $\chi^{2}$ of the data and the model, are shown in Fig. 2 as continuous red lines. The function used to analyze the inelastic spectra is the one of Eq. (10) convoluted with a square function approximating the instrumental resolution in $q$ space, with a width $\Delta q \sim$ $0.32 \mathrm{~nm}^{-1}$, a quantity almost negligible given the $q$ range in Fig. 2. 


\section{RESULTS}

As shown in Fig. 2, the curves well describe the spectra at low frequencies, apart from the high $q$ points $\left(q>10 \mathrm{~nm}^{-1}\right)$. At higher frequencies also the low $q$ range $\left(q<2 \mathrm{~nm}^{-1}\right)$ is not well reproduced. However, the reasonable agreement between the model and the data requires $A$ to be greater than one in the entire explored frequency range. Such a value of $A$ is of difficult interpretation within the theoretical models discussed in the introduction. We will propose an explanation for the behavior of the parameter $A$ in the discussion section.

Now we show the results of the fitting procedure with the constraint: $A=1$. The corresponding curves are plotted as dashed lines in Fig. 2. Apart from the lower-frequency spectra, which are well reproduced, the model systematically underestimates the spectra at all other frequencies. Having analyzed the experimental spectra both at a fixed $q$ as a function of frequency, by means of a single DHO, and as a function of the wave vector at a fixed $\omega$, by means of the model of Eq. (10), we can now compare the results from the two approaches.

The peak position gives the apparent sound velocity $\mathrm{v}=$ $\sqrt{\Sigma^{\prime}(\omega)}$, a quantity similar to the one determined from the DHO model: $\mathrm{v}_{\mathrm{DHO}}=\Omega(q) / q$. In order to compare the two quantities we have to translate the DHO parameters $(\Omega, \Gamma, f, S)$ to the frequency domain, by assuming $\omega_{\text {DHO }}=\Omega(q)$. In Fig. 3 we plot the DHO parameters corresponding to $q<5 \mathrm{~nm}^{-1}$, because in this range the dispersion curve is monotonic, at variance with the higher- $q$ behavior that will be discussed in the next section. As shown in the top panel of the figure, the sound velocity is marked by a softening close to the frequency of the BP (around $6 \mathrm{meV}$ at this temperature), already reported in Ref. [15], followed by a positive dispersion at higher frequencies. No noticeable difference is observed between the results of the model of Eq. (10) with free $A$ (dots) and with $A=1$ (triangles). Also $\mathrm{v}_{\mathrm{DHO}}$ is close to $\mathrm{v}$ in the entire range and shows a similar trend.

The bottom panel of Fig. 3 shows the comparison between the quantity $\Sigma^{\prime \prime}(\omega) / \omega$ and $\Gamma(q) / q^{2}$ of the DHO [see Eq. (8)]. More precisely, the plotted quantity for the $\Sigma=\Sigma(\omega)$ model is $\Sigma^{\prime \prime} / \omega-\Gamma_{\mathrm{RES}} \Sigma^{\prime} / \omega^{2}$, where $\hbar \Gamma_{\mathrm{RES}} \sim 1.5 \mathrm{meV}$ is the full width at half maximum of the instrumental resolution function $R(\omega)$. Below the BP frequency the plotted quantity follows the Rayleigh scattering law and is thus proportional to the square of the frequency. In this regime the three models give similar results. Above the BP frequency this quantity becomes essentially frequency independent, although some quantitative differences are observed between the three approaches. Specifically, the DHO and the $q$-space model with free $A$ give similar results at almost all frequencies, with differences in the width only in an intermediate range, while the model with $A=1$ has values a factor of two smaller at high $\omega$, a difference already discernible from the spectra in Fig. 2.

Finally, we compare the parameter $A(\omega)$ with the corresponding quantity for the DHO model. The inelastic part of the dynamic structure factor for the DHO can be written as:

$$
\begin{aligned}
& S_{\text {inel,DHO }}^{c l}(q, \omega) \\
& \quad=S(q)[1-f(q)] \frac{1}{\pi} \frac{\Omega^{2}(q) \Gamma(q)}{\left[\omega^{2}-\Omega^{2}(q)\right]^{2}+\omega^{2} \Gamma^{2}(q)} .
\end{aligned}
$$
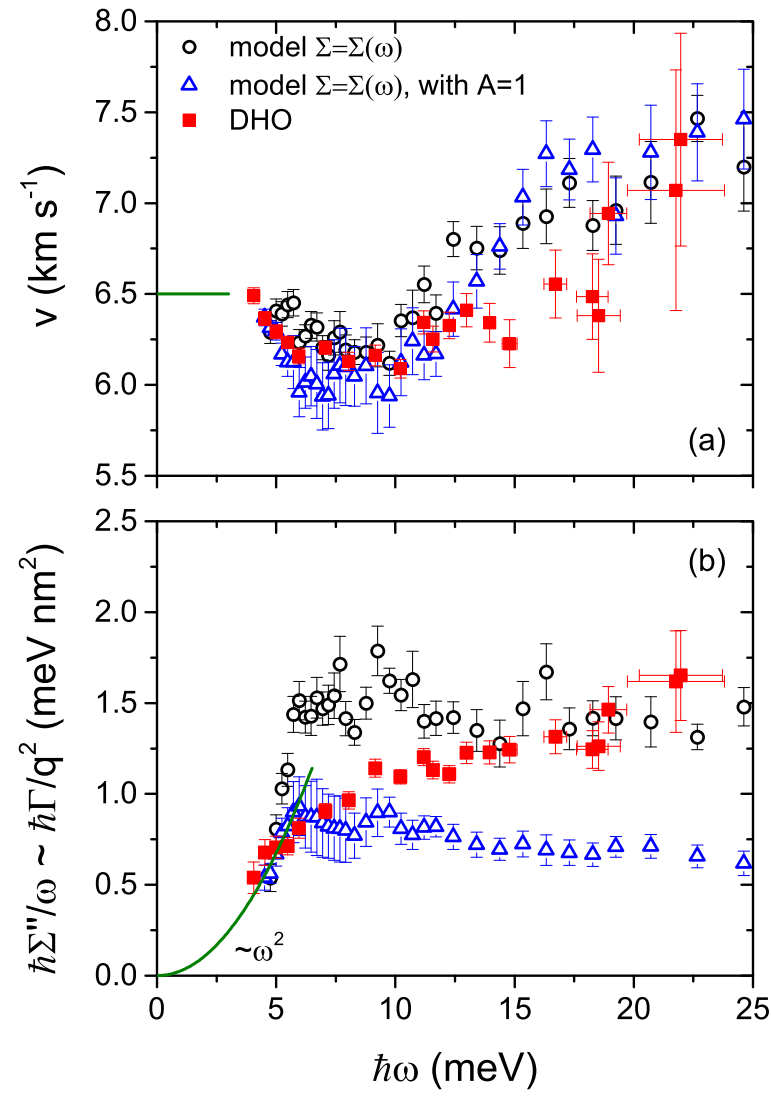

FIG. 3. (a) Sound velocity as a function of frequency for the model with $q$-independent self-energy with free $A$ (black circles) and with $A=1$ (blue triangles) and for the DHO model (red squares). The continuous (green) line is the value of the macroscopic longitudinal sound velocity at the temperature of the experiment. (b) Sound attenuation divided by $q^{2}$ as a function of frequency. Same symbols as in (a).

In this expression the frequency normalization is explicit, since $S(q)[1-f(q)]$ is the frequency integral of $S_{\text {inel,DHO. }}^{c l}$. Comparing Eq. (11) with expressions (6), (7), and (8), we find that the DHO model can be expressed in terms of the set of parameters $\left(\Omega, \Gamma, f, A_{\mathrm{DHO}}\right)$, instead of the parameters $(\Omega, \Gamma, f, S)$, by defining $A_{\mathrm{DHO}}$ as the zeroth moment of the classical current correlation function in units of the square of the thermal velocity ${ }^{1}$ :

$$
\begin{aligned}
A_{\mathrm{DHO}} & =\frac{m}{k_{B} T} \int_{-\infty}^{\infty} d \omega \frac{\omega^{2}}{q^{2}} S_{\mathrm{DHO}}^{c l}(q, \omega) \\
& =\frac{m}{k_{B} T} S(q)[1-f(q)] \frac{\Omega^{2}(q)}{q^{2}} .
\end{aligned}
$$

Note that a value of $A$ different from 1 implies a violation of the classical second moment sum rule. Such a violation is indeed found for both the DHO model and the model with a $q$-independent self energy, as shown in Fig. 4. Here, as in Fig. 3, the parameter of the DHO is plotted as a function of

\footnotetext{
${ }^{1}$ We assume, as in Eq. (6), that the elastic part of the dynamic structure factor is proportional to $\delta(\omega)$.
} 


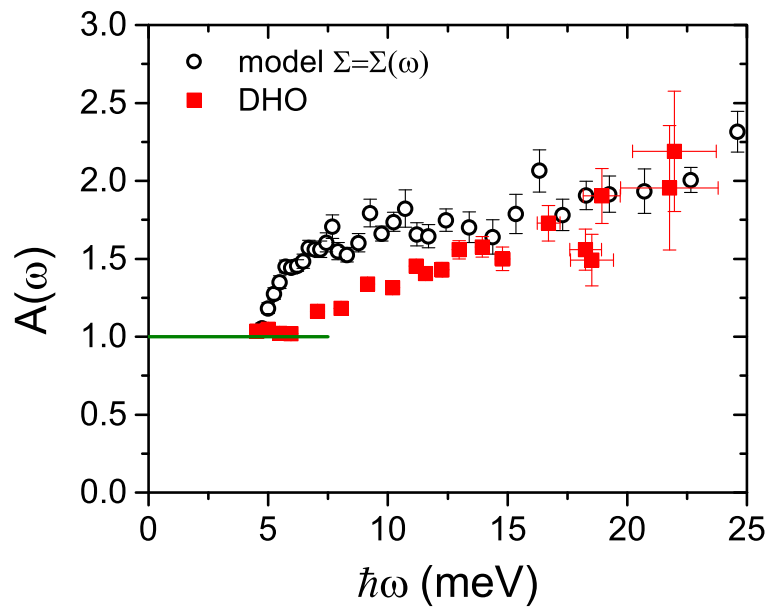

FIG. 4. Parameter $A=A(\omega)$ for the model with $q$-independent self-energy (black circles) and for the DHO model (red squares). The line is set at $A=1$.

$\hbar \Omega(q)$, whose uncertainty is evidenced in the horizontal error bars. Only the first few points of the DHO model, those below the BP frequency, are consistent with a value of $A$ close to one.

At the highest frequencies both models give similar values for the parameter $A$, while the discrepancy in the range between $5 \mathrm{meV}$ and $15 \mathrm{meV}$ is related to the fact that the comparison is done in different regions of the $(q, \omega)$ space. Specifically, the $\Sigma=\Sigma(\omega)$ model is fitted to the entire measured $q$ range (between 1 and $12 \mathrm{~nm}^{-1}$ ), while the DHO parameters in this frequency interval correspond to $q$ between 1 and $4 \mathrm{~nm}^{-1}$. It will be clear from the discussion in the next section that the high- $q$ tail of the $\Sigma=\Sigma(\omega)$ model (see Fig. 2) is picking up intensity from vibrational modes that appear in the DHO analysis as peaked at $\sim 10 \mathrm{meV}$ for $q>5 \mathrm{~nm}^{-1}$. This additional intensity gives rise to an increase of the parameter $A$ over the one of the DHO, as shown in Fig. 4, and induces an additional broadening of the peak, as observed in Fig. 3(b).

\section{DISCUSSION}

We come to the conclusion that both models, the one based on the assumption of a $q$-independent self-energy and the $\mathrm{DHO}$, violate the classical second moment sum rule above the frequency of the BP. In the case of $\mathrm{SiO}_{2}$ this is also the upper frequency where the damping follows the Rayleigh scattering law, as shown in Fig. 3(b). Based on a previous work where we compared the vibrational dynamics of a permanently densified $\mathrm{SiO}_{2}$ sample with that of an $\alpha$-quartz polycrystal [24], we are led to believe that this is the upper frequency where a description of the dynamics in terms of a single excitation is still appropriate, as also found for other glasses [33]. The use of a single-excitation model is probably the reason for the violation of the classical second moment sum rule.

Consequently, the sum rule has to be used with care if one wants to exploit its validity to determine the $q$ dependence of the spectra. For instance, we expect the procedure followed by Buchenau [22] to fail at high frequencies, above the BP, since he applies the classical second moment sum rule to a single DHO model. Buchenau also suggests that at high $q$ s a part of the inelastic intensity is due to diffuse umklapp scattering. In the case of our measurements this contribution is probably relevant only for the points above $20 \mathrm{~nm}^{-1}$, see Fig. 1, and possibly also for the highest $q$ points of Fig. 2 . The inelastic intensity at such high $q$ s closely resembles the density of vibrational states and is dominated by the transverse acousticlike branch at the zone boundary [24].

To clarify the origin of the deviation of the parameter $A$ from unity, it is useful to look at the $q$ dependence of the parameters of the DHO model and to compare the single peak analysis with previous studies [32,34] where the spectra were fitted with a double-excitation model. The dispersion curve and the apparent sound velocity obtained from these two analyses and the parameter $A_{\mathrm{DHO}}$ are plotted in Fig. 5. The first panel of the figure shows that the peak of the single DHO follows the higher-frequency mode of the double excitation model up to $5 \mathrm{~nm}^{-1}$. At higher $q$ s the single DHO is peaked on the lower-frequency excitation. This transition is quite abrupt and is related to the increase in intensity of the lower-frequency peak, which overcomes that of the higher-frequency one in this wave-vector range, as found in Ref. [32]. The shift of the single DHO from one peak to the other is probably accelerated by the limited energy interval, $\pm 40 \mathrm{meV}$, where the $1620 \mathrm{~K}$ data have been measured.

This change of behavior of the single DHO model for $q \sim 5 \mathrm{~nm}^{-1}$ is reflected also in the $q$ dependence of the parameter $A_{\mathrm{DHO}}$, which increases almost linearly with $q$ in the range where the apparent sound velocity is approximately constant, while it drops rapidly towards zero at higher $q s$. The initial increase of the parameter $A_{\mathrm{DHO}}$ can be ascribed to the slow decay of the tails of the single DHO model. The shape of the function leads to overestimate the area of the current correlation function in the frequency range outside of the measured one. The subsequent rapid decrease of $A_{\mathrm{DHO}}$ is due to the fact that, for $q>5 \mathrm{~nm}^{-1}$, the single DHO describes only a portion of the spectrum. This part of the spectrum, despite being predominant in the $S(q, \omega)$, has a negligible intensity in the current correlation function with respect to higher-frequency components.

It is interesting to note that the single DHO model is always peaked in a frequency range mainly populated by acousticlike excitations, as can be seen by comparing our analysis of amorphous silica with that of the corresponding crystal, $\alpha$-cristobalite. In a recent experimental and numerical work, Weingher et al. [35] have determined with high accuracy the dispersion curves of a single crystal of $\alpha$-cristobalite, which belongs to the tetragonal lattice system. The curves are plotted along a few high symmetry directions in Fig. 6 . The red squares in the figure are the frequencies of the single DHO model for supercooled silica at the $q$ values which equal the modulus of the wave vector in the given crystallographic direction. Note that in this representation some data of amorphous silica are reported more than once, since different wave vectors in the chosen directions of the first Brillouin zone have the same modulus.

It is worth noting that the acoustic modes of the single crystal are restricted to energies below $18 \mathrm{meV}$. Comparing the dispersion curves of the crystal with those for the glass, we see that the lower-frequency peak of the double-excitation model of Fig. 5 is not the transverse acoustic mode, apart possibly 

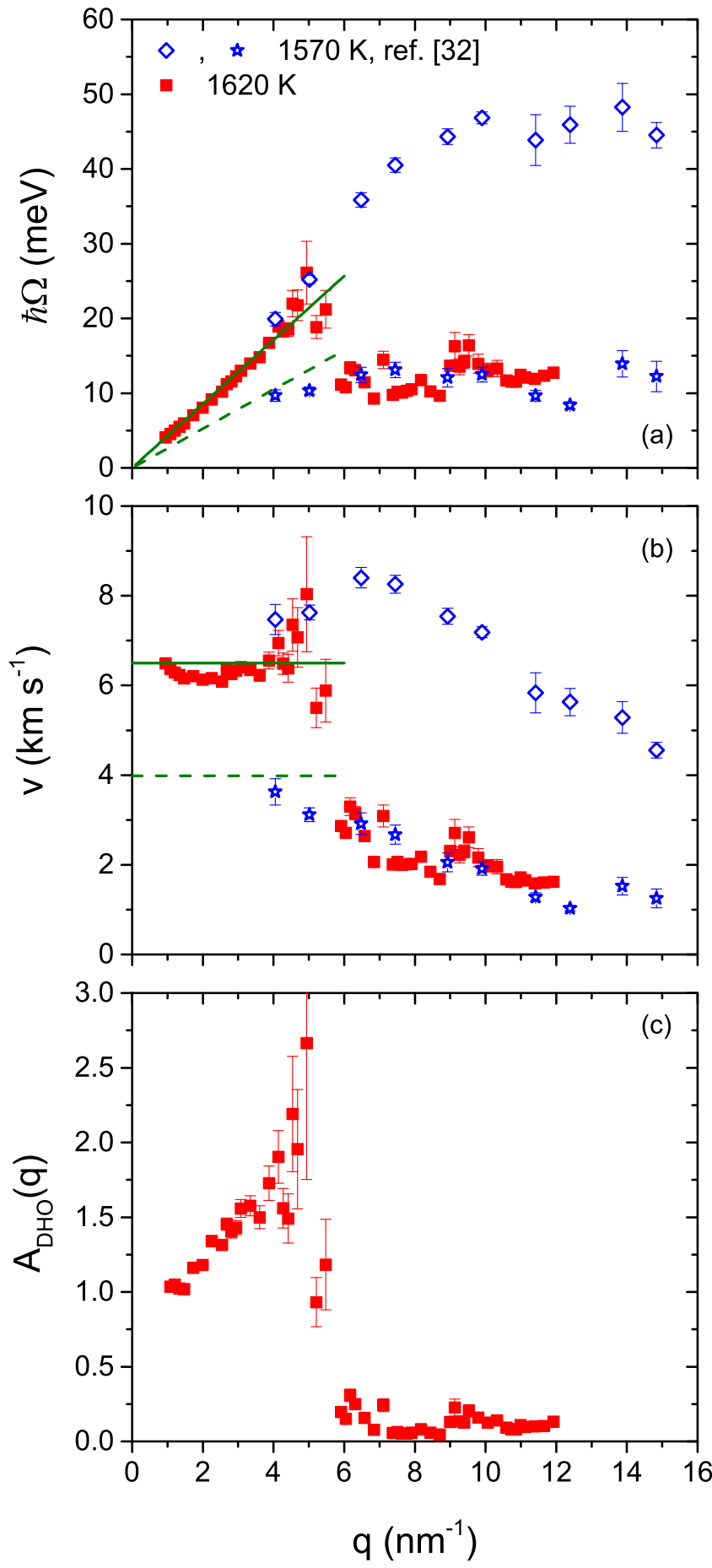

FIG. 5. Wave-vector dependence of the parameters of the single DHO model (red squares) and comparison with a previous experiment [32] at a similar temperature $(1570 \mathrm{~K})$ analyzed with a two excitations model (open blue diamonds for the higher-frequency mode and open blue stars for the lower-frequency one). The lines correspond to the macroscopic longitudinal (continuous green) and transverse (dashed green) sound velocity. (a) Dispersion curve; (b) apparent sound velocity; (c) parameter A for the single DHO model.

from the first few points, but rather a peak with contributions from all the three acoustic branches and also from the first optic branch. Similarly, the high-frequency peak of the two excitations model for the glass is not the high- $q$ counterpart of

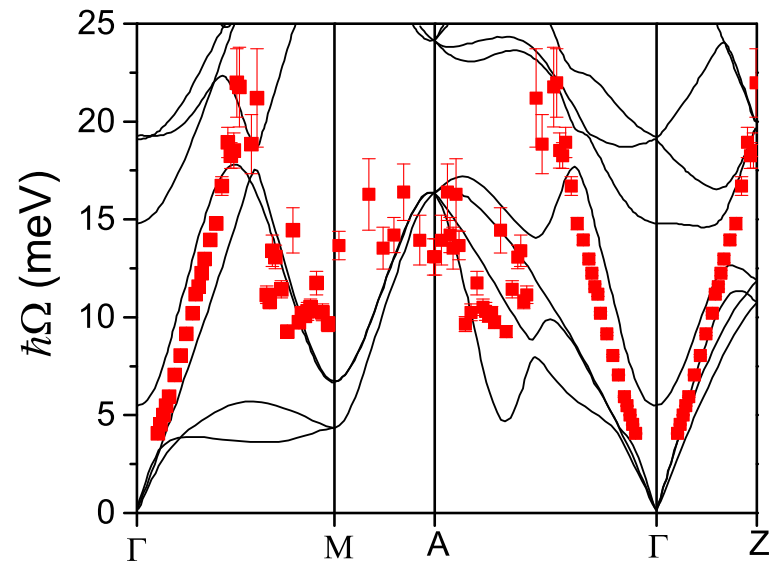

FIG. 6. Dispersion curves of $\alpha$-cristobalite along a few high symmetry directions (black lines) from Ref. [35], compared to the dispersion of the single DHO model for supercooled silica (red squares) as described in the text. For reference, the lengths of the plotted directions in the Brillouin zone are: $\overline{\Gamma M} \sim 9.0 \mathrm{~nm}^{-1}$, $\overline{\Gamma A} \sim 10.1 \mathrm{~nm}^{-1}$, and $\overline{\Gamma Z} \sim 4.6 \mathrm{~nm}^{-1}$.

the longitudinal acoustic mode but, above $5 \mathrm{~nm}^{-1}$, that peak is probably dominated by opticlike excitations.

\section{CONCLUSIONS}

We have presented an analysis of IXS data of vitreous silica in the supercooled liquid state $(T=1620 \mathrm{~K})$ performed in wave-vector space. We have applied a careful normalization procedure to convert the experimental spectra, measured at fixed $q$ as a function of frequency, to a set of $q$-dependent spectra at fixed $\omega$. The resulting data have been modeled in terms of a $q$-independent complex self-energy, $\Sigma=\Sigma(\omega)$, in order to test the validity of recent theories for the vibrations in glasses [18-21]. The analysis has revealed that such an approach can be used only at the price of including a frequencydependent constant, $A(\omega)$, to multiply the inelastic intensity. The comparison with the experimental data shows that this parameter is different from one in the entire explored frequency range, leading to a violation of the classical second moment sum rule. The agreement between the model and the spectra is far less satisfying if one imposes $A=1$, apart from the first few points at low frequencies, close and below the frequency of the BP ( $\hbar \omega_{B P} \sim 6 \mathrm{meV}$ in supercooled silica).

The knowledge of both the $\omega$ and $q$ dependence of the experimental dynamic structure factor allowed us to determine the inelastic intensity of a single DHO model in absolute units. To this aim we extended the previously published analysis [15], that was limited to $q<4 \mathrm{~nm}^{-1}$, to the entire measured $q$ range. The comparison between the single DHO model and the model with a $q$-independent self-energy shows that both give similar parameters and in both cases the classical second moment sum rule is violated above the frequency of the BP. The reason for this failure lies in the fact that a single-excitation model cannot appropriately describe the spectra at high frequencies, where the overlap of many vibrational modes gives rise to a complex spectral shape.

The comparison of the single DHO model with the dynamics of $\alpha$-cristobalite single crystal indicates that the 
structure of the glass on the medium range cannot be neglected in a proper description of its vibrational dynamics. The theories based on a $q$-independent self-energy are, instead, assuming an almost linear dispersion with a $q$-independent sound velocity, thus neglecting the effect of the finite size of the Brillouin zone $[33,36]$.

To conclude, single-excitation models with a $q$-independent self-energy are able to describe the experimental data only below the BP frequency. In the case of vitreous silica, this is also the upper frequency where Rayleigh scattering is observed. The Rayleigh scattering can be modeled quantitatively by assuming the elastic constants to be correlated on a length scale comparable to the extent of the medium range order of the glass [17,24]. We suggest that these scattering centers can be identified with the elastic heterogeneities observed in the numerical simulation studies [4-9]. At higher frequencies the vibrational dynamics of the glass closely resembles that of the corresponding polycrystal and the $\mathrm{BP}$ lies close to the first Van Hove singularity of the crystal [23-25,35].
[1] L. Berthier, G. Biroli, J.-P. Bouchaud, L. Cipelletti, D. El Masri, D. L'Hôte, F. Ladieu, and M. Pierno, Science 310, 1797 (2005).

[2] T. Scopigno, G. Ruocco, F. Sette, and G. Monaco, Science 302, 849 (2003).

[3] V. N. Novikov and A. P. Sokolov, Nature (London) 431, 961 (2004).

[4] A. Tanguy, J. P. Wittmer, F. Léonforte, and J.-L. Barrat, Phys. Rev. B 66, 174205 (2002).

[5] F. Léonforte, A. Tanguy, J. P. Wittmer, and J.-L. Barrat, Phys. Rev. Lett. 97, 055501 (2006).

[6] K. Yoshimoto, T. S. Jain, K. Van Workum, P. F. Nealey, and J. J. de Pablo, Phys. Rev. Lett. 93, 175501 (2004).

[7] R. A. Riggleman, J. F. Douglas, and J. J. de Pablo, Soft Matter 6, 292 (2010).

[8] H. Mizuno, S. Mossa, and J.-L. Barrat, Phys. Rev. E 87, 042306 (2013).

[9] H. Mizuno, S. Mossa, and J.-L. Barrat, Proc. Natl. Acad. Sci. USA 111, 11949 (2014).

[10] H. Wagner, D. Bedorf, S. Küchemann, M. Schwabe, B. Zhang, W. Arnold, and K. Samwer, Nat. Mater. 10, 439 (2011).

[11] Y. Luo, Q.-K. Li, and M. Li, J. Appl. Phys. 117, 044301 (2015).

[12] B. Rufflé, M. Foret, E. Courtens, R. Vacher, and G. Monaco, Phys. Rev. Lett. 90, 095502 (2003).

[13] B. Rufflé, G. Guimbretière, E. Courtens, R. Vacher, and G. Monaco, Phys. Rev. Lett. 96, 045502 (2006).

[14] G. Monaco and V. M. Giordano, Proc. Natl. Acad. Sci. USA 106, 3659 (2009).

[15] G. Baldi, V. M. Giordano, G. Monaco, and B. Ruta, Phys. Rev. Lett. 104, 195501 (2010).

[16] G. Baldi, V. M. Giordano, and G. Monaco, Phys. Rev. B 83, 174203 (2011).

[17] G. Baldi, V. M. Giordano, B. Ruta, R. Dal Maschio, A. Fontana, and G. Monaco, Phys. Rev. Lett. 112, 125502 (2014).

[18] W. Schirmacher, G. Ruocco, and T. Scopigno, Phys. Rev. Lett. 98, 025501 (2007).

[19] A. Marruzzo, W. Schirmacher, A. Fratalocchi, and G. Ruocco, Sci. Rep. 3, 1407 (2013).

[20] E. DeGiuli, A. Laversanne-Finot, G. Düring, E. Lerner, and M. Wyart, Soft Matter 10, 5628 (2014).

[21] E. DeGiuli, E. Lerner, C. Brito, and M. Wyart, Proc. Natl. Acad. Sci. USA 111, 17054 (2014).
[22] U. Buchenau, Phys. Rev. E 90, 062319 (2014).

[23] A. I. Chumakov, G. Monaco, A. Monaco, W. A. Crichton, A. Bosak, R. Rüffer, A. Meyer, F. Kargl, L. Comez, D. Fioretto, H. Giefers, S. Roitsch, G. Wortmann, M. H. Manghnani, A. Hushur, Q. Williams, J. Balogh, K. Parliński, P. Jochym, and P. Piekarz, Phys. Rev. Lett. 106, 225501 (2011).

[24] G. Baldi, M. Zanatta, E. Gilioli, V. Milman, K. Refson, B. Wehinger, B. Winkler, A. Fontana, and G. Monaco, Phys. Rev. Lett. 110, 185503 (2013).

[25] A. I. Chumakov, G. Monaco, A. Fontana, A. Bosak, R. P. Hermann, D. Bessas, B. Wehinger, W. A. Crichton, M. Krisch, R. Rüffer, G. Baldi, G. Carini, G. Carini, G. D’Angelo, E. Gilioli, G. Tripodo, M. Zanatta, B. Winkler, V. Milman, K. Refson, M. T. Dove, N. Dubrovinskaia, L. Dubrovinsky, R. Keding, and Y. Z. Yue, Phys. Rev. Lett. 112, 025502 (2014).

[26] O. Pilla, A. Cunsolo, A. Fontana, C. Masciovecchio, G. Monaco, M. Montagna, G. Ruocco, T. Scopigno, and F. Sette, Phys. Rev. Lett. 85, 2136 (2000).

[27] T. Scopigno, U. Balucani, G. Ruocco, and F. Sette, J. Phys.: Condens. Matter 12, 8009 (2000).

[28] V. M. Giordano and G. Monaco, J. Chem. Phys. 131, 014501 (2009).

[29] B. Ruta, G. Monaco, V. M. Giordano, F. Scarponi, D. Fioretto, G. Ruocco, K. S. Andrikopoulos, and S. N. Yannopoulos, J. Phys. Chem. B 115, 14052 (2011).

[30] B. Ruta, G. Baldi, F. Scarponi, D. Fioretto, V. M. Giordano, and G. Monaco, J. Chem. Phys. 137, 214502 (2012).

[31] R. L. Mozzi and B. E. Warren, J. Appl. Crystallogr. 2, 164 (1969).

[32] G. Baldi, V. M. Giordano, G. Monaco, F. Sette, E. Fabiani, A. Fontana, and G. Ruocco, Phys. Rev. B 77, 214309 (2008).

[33] L. Orsingher, G. Baldi, A. Fontana, L. E. Bove, T. Unruh, A. Orecchini, C. Petrillo, N. Violini, and F. Sacchetti, Phys. Rev. B 82, 115201 (2010).

[34] B. Ruzicka, T. Scopigno, S. Caponi, A. Fontana, O. Pilla, P. Giura, G. Monaco, E. Pontecorvo, G. Ruocco, and F. Sette, Phys. Rev. B 69, 100201 (2004).

[35] B. Wehinger, A. Bosak, K. Refson, A. Mirone, A. Chumakov, and M. Krisch, J. Phys.: Condens. Matter 27, 305401 (2015).

[36] P. Benassi, M. Nardone, A. Giugni, G. Baldi, and A. Fontana, Phys. Rev. B 92, 104203 (2015). 